\title{
Modification des équations d'état pour une meilleure prédiction des propriétés thermodynamiques des pétroles bruts
}

\author{
S. Sator ${ }^{1}$ et C.E. Chitour ${ }^{1}$ \\ 1 Laboratoire de valorisation des énergies fossiles, Département de génie chimique, École nationale polytechnique, \\ 10, avenue Hassen Badi, El Harrach, Alger - Algérie \\ e-mail :
}

Résumé - Ce travail consiste à proposer deux modifications des équations d'état cubiques pour une meilleure prédiction des propriétés thermodynamiques des pétroles bruts. L'élaboration de ces nouvelles équations d'état est basée sur deux approches alternatives.

La première approche a consisté à représenter le paramètre Alpha des équations d'état cubiques selon une forme généralisée. Dans la seconde approche, ce même paramètre Alpha a été modifié selon une procédure similaire à celle adoptée par Soave (1993).

Les équations d'état établies à partir des données des propriétés des $n$-alcanes se sont avérées efficaces dans la prédiction des propriétés thermodynamiques d'autres familles d'hydrocarbures.

L'application de ces équations d'état aux mélanges et aux fractions pétrolières a donné des résultats très satisfaisants comparativement aux autres équations d'état de la littérature.

Mots-clés : équations d'état, propriétés thermodynamiques, pétrole brut, prédiction.

Abstract - Equations of State Modifications for a Better Prediction of Thermodynamic Properties of Crude Oils - This work consists of two modifications of cubic equations of state for a better prediction of the crude oils thermodynamic properties. The development of these new equations of state is based on two alternative approaches.

The first approach consisted in the representation of Alpha cubic equations of state parameter in a generalised form. In the second, the same parameter was modified by a procedure which is similar to Soave's (1993).

The equations obtained were established from $\mathrm{n}$-alkanes properties data and proved effective in the prediction of the thermodynamic properties of other hydrocarbon families.

The application of these equations to mixtures and oil fractions gave very satisfying results compared to other equations of state in the literature.

Keywords: equations of state, thermodynamic properties, crude oil, prediction. 


\section{NOTATIONS}

$\Delta H^{\text {vap }} \quad$ enthalpie de vaporisation

$\Delta S^{\text {vap }} \quad$ entropie de vaporisation

$\mathrm{HC}$ hydrocarbure

$M \quad$ masse molaire

$P \quad$ pression

$P_{b} \quad$ pression de bulle

$P_{c} \quad$ pression critique

PR équation d'état de Peng-Robinson

PRG1 équation d'état de Peng-Robinson généralisée par Sator-Chitour

PRG2 équation d'état de Peng-Robinson généralisée dans cette étude

PRM équation d'état de Peng-Robinson modifiée

$\mathrm{R} \quad$ constante des gaz parfaits

RK équation d'état de Redlich-Kwong

RKG1 équation d'état de Redlich-Kwong généralisée par Sator-Chitour

RKG2 équation d'état de Redlich-Kwong généralisée dans cette étude

RKM équation d'état de Redlich-Kwong modifiée

SRK équation d'état de Soave-Redlich-Kwong

SVDW équation d'état de Soave-van der Waals

$T \quad$ température

$T_{c} \quad$ température critique

TPR équation d'état de Twu-Peng-Robinson

TRK équation d'état de Twu-Redlich-Kwong

$T_{r} \quad$ température réduite

$V \quad$ volume

$V^{L} \quad$ volume spécifique de la phase liquide

$V^{V} \quad$ volume spécifique de la phase vapeur

VDW équation d'état de van der Waals

VDWG1 équation d'état de van der Waals généralisée par Sator-Chitour

VDWG2 équation d'état de van der Waals généralisée dans cette étude

$Z R A$

facteur de compressibilité de Rackett

\section{Lettres grecques}

$\alpha \quad$ paramètre Alpha des équations d'état de RedlichKwong et Peng-Robinson

$\omega$ facteur acentrique

\section{INTRODUCTION}

De nombreux chercheurs ont proposé différentes méthodes d'estimation des propriétés thermodynamiques des corps purs, des mélanges et des fractions pétrolières par application des équations d'état, ou encore en utilisant des corrélations empiriques.
La première équation d'état cubique a été proposée par van der Waals (1873) :

$$
P=\mathrm{R} T /(V-b)-a / V^{2}
$$

avec :

$$
\begin{gathered}
a=0,42748\left(\mathrm{R} T_{c}\right)^{2} / P_{c} \\
b=0,125 \mathrm{R} T_{c} / P_{c}
\end{gathered}
$$

Elle a été suivie par de nombreuses autres équations d'état, dont celle de Redlich et Kwong (1949) qui a permis une représentation plus satisfaisante du comportement réel, particulièrement de la phase vapeur.

Soave (1972) a permis d'obtenir une équation applicable tout le long de la courbe de saturation, par l'intermédiaire d'un terme correctif «Alpha» qui tient compte non seulement de l'influence de la température mais aussi de la nature du fluide par l'intermédiaire du facteur acentrique $(\omega)$. Cependant, la prédiction de la densité liquide par cette équation d'état s'avère incorrecte. Pour pallier cela, Peng et Robinson (1976) ont amélioré l'équation de Soave en modifiant le paramètre Alpha. Ceci a permis d'obtenir des écarts pour la densité liquide variant entre $5 \%$ pour des températures réduites inférieures à 0,65 et $15 \%$ au voisinage du point critique.

Par la suite, de nombreuses équations ont été proposées. Parmi elles, nous pouvons citer l'équation à quatre paramètres de Jan-Tsai (Jan et Tsai, 1991). Twu et al. (1995) proposèrent d'exprimer la fonction Alpha des équations d'état de Redlich-Kwong et Peng-Robinson en fonction de la température réduite $\left(T_{r}\right)$ et du facteur acentrique $(\omega)$ de la manière suivante :

$$
\begin{gathered}
\alpha=\alpha^{(0)}+\omega\left(\alpha^{(1)}-\alpha^{(0)}\right) \\
\alpha^{(0)(1)}=T_{r}^{*} \mathrm{e}^{M\left(1-T_{r}^{0,5}\right)}
\end{gathered}
$$

La fonction Alpha étant linéaire en fonction du facteur acentrique, l'application de ces équations aux hydrocarbures lourds, aux fractions pétrolières et aux condensats est recommandée.

En se basant sur ces mêmes considérations, Sator et Chitour (1998) ont établi, en se basant sur la loi des états correspondants, une nouvelle fonction Alpha pour les équations d'état de Redlich-Kwong, Peng-Robinson et van der Waals, de la forme :

$$
\alpha=\alpha^{(0)}+\omega\left(\alpha^{(1)}-\alpha^{(0)}\right)
$$

$\alpha^{(0)(1)}=1+$

$$
\left[a\left(1-T_{r}\right)+b\left(1-T_{r}\right)^{1,5}+c\left(1-T_{r}\right)^{3}+d\left(1-T_{r}\right)^{6}\right] / T_{r}
$$

Le modèle mathématique de la fonction Alpha est une équation de type Wagner (1973). 


\section{1 ÉQUATIONS D'ÉTAT GÉNÉRALISÉES}

\section{1 Élaboration}

L'objectif de cette première partie de l'étude a consisté à exprimer la fonction Alpha selon la loi des états correspondants en modifiant la forme mathématique des fonctions $\alpha^{(0)}$ et $\alpha^{(1)}$ pour les équations de type Redlich-Kwong (RK), Peng-Robinson (PR) et van der Waals (VDW). Pour cela, nous avons pris en considération les $n$-alcanes allant du méthane au $n$-eicosane. Les propriétés critiques et les facteurs acentriques de ces hydrocarbures ont été recueillis dans la littérature à partir de la base de données du Design Institute for Physical Properties Data (DIPPR, 1995) et de celle donnée par Reid et al. (1988). Sur la base de ces données, nous avons calculé les valeurs d'Alpha pour des températures réduites comprises entre 0,3 et 1,0 avec un incrément de 0,05 pour chaque hydrocarbure étudié. Les données de tension de vapeur ont été calculées quant à elles en utilisant l'équation de Twu et al. (1994).

En représentant graphiquement Alpha en fonction du facteur acentrique à des valeurs de température réduite constantes, nous vérifions dans la figure 1 l'hypothèse de linéarité. À partir des pentes et des ordonnées à l'origine des droites obtenues à différentes valeurs de $T_{r}$, nous avons pu déduire les valeurs de $\alpha^{(0)}$ et $\alpha^{(1)}$. Nous avons par la suite procédé à une modélisation de ces deux paramètres selon la forme :

$\alpha^{(0)(1)}=1+$

$$
\left[a\left(1-T_{r}\right)+b\left(1-T_{r}\right)^{2}+c\left(1-T_{r}\right)^{4}+d\left(1-T_{r}\right)^{6}\right] / T_{r}
$$

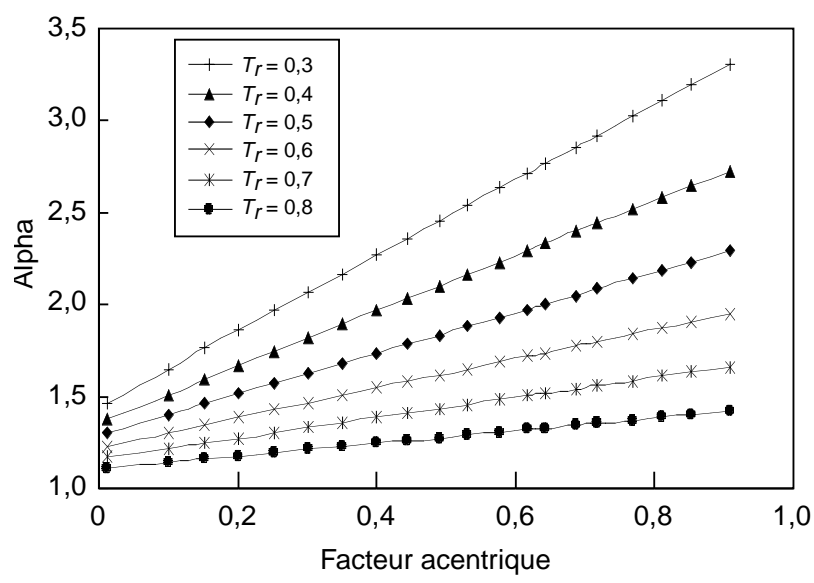

Figure 1

Variation d'Alpha en fonction du facteur acentrique à $T_{r}$ constante pour l'équation de Redlich-Kwong.

Variation of Alpha versus acentric factor at constant $T_{r}$ for the Redlich-Kwong equation.
Notons que cette forme mathématique vérifie bien la condition au point critique $\alpha=1,0$ quand $T_{r}=1,0$. Les paramètres $a, b, c$ et $d$ pour les trois équations d'état élaborées, van der Waals généralisée (VDWG2), Redlich-Kwong généralisée (RKG2) et Peng-Robinson généralisée (PRG2), sont donnés dans le tableau 1.

\subsection{Application aux hydrocarbures purs}

La fonction Alpha étant initialement proposée afin de réajuster l'équation d'état aux données de tension de vapeur, nous considérons cette propriété comme la plus significative quant à la validité de toute équation d'état. Ainsi, nous avons calculé cette propriété pour cinq familles d'hydrocarbures (HC) : $n$-alcanes, alcanes ramifiés, alkylbenzènes, alkylcyclopentanes et alkylcyclohexanes, en utilisant les équations établies et celles proposées par d'autres chercheurs, à savoir les équations d'état généralisées de van der Waals (VDWG1), Redlich-Kwong (RKG1) et Peng-Robinson (PRG1) établies par Sator et Chitour (1998), ainsi que celles de Twu-RedlichKwong (TRK) (Twu et al., 1995), Twu-Peng-Robinson (TPR) (Twu et al., 1995) et Soave-van der Waals (SVDW) (Soave, 1984).

Les écarts absolus enregistrés par les différentes équations par rapport aux valeurs de référence calculées par les équations recommandées par Reid et al. (1988) ou par le DIPPR (1995) sont donnés dans le tableau 2. En général, on note une amélioration des écarts pour les trois équations élaborées et ce, par rapport aux autres équations d'état, exception faite des équations de Sator-Chitour qui ont enregistré des écarts légèrement plus faibles. Enfin, bien qu'ayant été établies à partir des données des $n$-alcanes, les nouvelles équations d'état donnent de bons résultats pour les autres familles d'hydrocarbures, ce qui est en bon accord avec le principe des états correspondants.

Nous avons procédé également au calcul de l'enthalpie de vaporisation $\left(\Delta H^{\text {vap }}\right)$, de l'entropie de vaporisation $\left(\Delta S^{\text {vap }}\right)$ et des volumes spécifiques liquide $\left(V^{L}\right)$ et vapeur $\left(V^{V}\right)$.

Les résultats obtenus ont été comparés dans le tableau 3 aux valeurs expérimentales recueillies à partir de la littérature : Perry et Chilton (1990) et Raznjevic (1976).

Dans l'ensemble, les équations d'état établies n'améliorent pas les écarts. Ces derniers restent du même ordre de grandeur que ceux obtenus par les équations d'état existantes.

\subsection{Application aux mélanges}

Nous avons appliqué les nouvelles équations au calcul de la pression de bulle $P_{b}$ et du volume spécifique liquide $V^{L}$ des mélanges binaires suivants : méthane-butane, méthanedécane et propane-pentane. Ces mélanges ont été étudiés par Sage et al. (1940). 


\section{TABLEAU 1}

Coefficients des paramètres $\alpha^{(0)}$ et $\alpha^{(1)}$ des équations d'état VDWG2, RKG2 et PRG2

Coefficients of $\alpha^{(0)}$ and $\alpha^{(1)}$ parameters of VDWG2, RKG2 and PRG2 equations of state

\begin{tabular}{c|c|c|c|c|c|c}
\cline { 2 - 7 } & \multicolumn{2}{c|}{ VDWG2 } & \multicolumn{2}{c}{ RKG2 } & \multicolumn{2}{c}{ PRG2 } \\
\cline { 2 - 7 } & $\alpha^{(0)}$ & $\alpha^{(1)}$ & $\alpha^{(0)}$ & $\alpha^{(1)}$ & $\alpha^{(1)}$ & 1,8175639 \\
$a$ & 0,55336656 & 2,12019860 & 0,52427642 & 2,069903 & 0,38925817 & 1,0723619 \\
$b$ & 0,51273619 & 1,42416660 & 0,46746036 & 1,3677951 & 0,28463341 & 0,02320135 \\
$c$ & 0,06084280 & 0,32219409 & 0,01264123 & 0,28383665 & 0,11048594 & 0,56124065 \\
$d$ & 0,099217475 & 0,84977938 & 0,05689029 & 0,81623559 & 0,03651989 & \\
\hline
\end{tabular}

TABLEAU 2

Écarts absolus en \% obtenus pour le calcul de la tension de vapeur

Absolute deviations in \% obtained for the calculated vapour pressure

\begin{tabular}{l|c|c|c|c|c|c|c|c|c|c|c|c|c}
\hline HC & Nombre & Points & VDWG1 & VDWG2 & SVDW & RKG1 & RKG2 & TRK & SRK & PRG1 & PRG2 & TPR & PR \\
\hline$n$-alcanes & 22 & 1334 & 1,85 & 1,84 & 3,69 & 1,80 & 1,85 & 2,45 & 2,80 & 1,85 & 1,98 & 2,42 & 11,72 \\
Alcanes ramifiés & 45 & 2164 & 1,30 & 1,34 & 2,41 & 1,23 & 1,34 & 1,57 & 1,89 & 1,29 & 1,37 & 1,63 & 2,26 \\
Alkylbenzènes & 29 & 1718 & 5,66 & 5,68 & 6,24 & 5,31 & 5,69 & 7,37 & 5,36 & 5,62 & 5,67 & 7,22 & 9,48 \\
Alkylcyclopentanes & 16 & 737 & 2,05 & 2,12 & 2,45 & 2,08 & 2,11 & 2,18 & 2,34 & 2,05 & 2,09 & 2,17 & 2,67 \\
Alkylcyclohexanes & 10 & 592 & 11,80 & 11,85 & 14,63 & 12,01 & 11,84 & 9,49 & 16,97 & 11,85 & 11,83 & 9,99 & 27,60 \\
Moyenne & 122 & 6545 & 3,59 & 3,62 & 4,79 & 3,49 & 3,62 & 4,06 & 4,40 & 3,58 & 3,65 & 4,08 & 8,42 \\
\hline
\end{tabular}

TABLEAU 3

Écarts absolus en \% obtenus pour le calcul des propriétés des corps purs Absolute deviations in \% obtained for the calculated properties of pure fluids

\begin{tabular}{l|c|c|c|c|c|c|c|c|c|c|c|c}
\hline Propriété & Points & SVDW & VDWG2 & VDWG1 & SRK & RKG2 & TRK & RKG1 & PR & PRG2 & TPR & PRG1 \\
\hline$\Delta H^{\text {vap }}$ & 277 & 2,48 & 1,87 & 1,65 & 2,28 & 1,85 & 1,96 & 1,65 & 1,88 & 1,82 & 1,94 & 1,72 \\
$\Delta S^{\text {vap }}$ & 269 & 2,66 & 2,10 & 1,98 & 2,49 & 2,12 & 2,24 & 1,93 & 1,96 & 2,09 & 2,21 & 1,94 \\
$V^{L}$ & 283 & - & - & - & 12,22 & 12,09 & 12,15 & 12,05 & 4,70 & 4,70 & 4,71 & 4,72 \\
$V^{V}$ & 247 & 3,62 & 3,71 & 3,76 & 2,48 & 2,52 & 2,41 & 2,51 & 3,11 & 2,21 & 2,27 & 1,98 \\
\hline
\end{tabular}

\section{TABLEAU 4}

Écarts absolus en \% obtenus pour le calcul des propriétés des mélanges

Absolute deviations in \% obtained for the calculated properties of mixtures

\begin{tabular}{|c|c|c|c|c|c|}
\hline Mélange & Propriété & SRK & SRKG2 & PR & PRG2 \\
\hline $\mathrm{C}_{1}-\mathrm{C}_{4}$ & $\begin{array}{c}P_{b} \\
V^{L} \\
V^{L} \text { translaté }\end{array}$ & $\begin{array}{l}13,58 \\
26,69 \\
29,84 \\
\end{array}$ & $\begin{array}{l}26,19 \\
14,05 \\
14,32\end{array}$ & $\begin{array}{c}13,99 \\
19,85 \\
- \\
\end{array}$ & $\begin{array}{c}5,21 \\
6,51 \\
- \\
\end{array}$ \\
\hline $\mathrm{C}_{1}-\mathrm{C}_{10}$ & $\begin{array}{c}P_{b} \\
V^{L} \\
V^{L} \text { translaté }\end{array}$ & $\begin{array}{l}16,04 \\
19,64 \\
17,84\end{array}$ & $\begin{array}{l}15,04 \\
18,56 \\
16,76\end{array}$ & $\begin{array}{c}17,11 \\
6,65 \\
-\end{array}$ & $\begin{array}{c}11,14 \\
6,21 \\
-\end{array}$ \\
\hline $\mathrm{C}_{3}-\mathrm{C}_{5}$ & $\begin{array}{c}P_{b} \\
V^{L} \\
V^{L} \text { translaté }\end{array}$ & $\begin{array}{c}2,52 \\
10,70 \\
10,33\end{array}$ & $\begin{array}{l}3,06 \\
9,81 \\
9,44\end{array}$ & $\begin{array}{c}3,35 \\
4,28 \\
-\end{array}$ & $\begin{array}{c}4,66 \\
4,28 \\
-\end{array}$ \\
\hline
\end{tabular}


En utilisant la règle de mélange conventionnelle de van der Waals et en considérant que les coefficients d'interaction binaires sont nuls puisqu'il s'agit de mélanges apolaires, nous avons appliqué les équations d'état généralisées au calcul de la pression de bulle et du volume spécifique liquide à différentes températures et pour différentes compositions correspondant à celles des points expérimentaux établis par Sage et al. (1940) : trente points expérimentaux pour les deux premiers mélanges et quinze points expérimentaux pour le troisième. Dans le cas des équations de type RK, nous avons de plus calculé le volume spécifique liquide par la translation de Péneloux et al. (1982), qui recommandent de soustraire au volume obtenu un volume $C$ donné par l'équation :

$$
C=0,407680(0,29441-Z R A) \mathrm{R} T_{c} / P_{c}
$$

où $Z R A$ est le facteur de compressibilité de Rackett, $\mathrm{R}$ la constante des gaz parfaits, et $T_{c}$ et $P_{c}$ respectivement la température et la pression critiques.

Les écarts absolus moyens enregistrés par rapport à l'expérience sont donnés dans le tableau 4.

Nous constatons, qu'en général, les équations proposées améliorent la prédiction de l'ensemble des propriétés étudiées, notamment l'équation PRG2 appliquée au premier mélange.

Dans le cas du mélange pentane-propane, les écarts obtenus sont relativement faibles par rapport aux deux autres mélanges, notamment pour la pression de bulle. Ceci est probablement dû au fait que les interactions entre le propane et le pentane ne sont pas aussi importantes que pour les autres compositions des deux autres mélanges.

Exemple de calcul de la pression de bulle et du volume spécifique liquide

Mélange méthane- $n$-butane contenant 8,5\% moles de méthane à $T=377,95 \mathrm{~K}$ par l'équation d'état RKG2 :

- $P_{\text {exp }}=35,98$ bar

- $V_{\text {exp }}^{L}=0,02279 \mathrm{~m}^{3} / \mathrm{kg}$

En prenant comme pression initiale la pression expérimentale, et après sept itérations successives, le processus a convergé et la pression de bulle est estimée alors à 43,157 bar, soit un écart absolu de 19,95\% par rapport à l'expérience.

Le volume spécifique liquide est quant à lui estimé à $2,2108710^{-3} \mathrm{~m}^{3} / \mathrm{kg}$, soit un écart absolu de $2,99 \%$.

La relation de Péneloux et al. (1982) permet ensuite de calculer un volume translaté de 2,20337 $10^{-3} \mathrm{~m}^{3} / \mathrm{kg}$, soit un écart absolu de 3,32\%.

\subsection{Application aux fractions pétrolières}

Des calculs de flash isotherme ont été réalisés sur deux fractions pétrolières de type naphta, commercialisées par Pan American Refining Co. et Humble Oil and Refining Co. Les données expérimentales concernant ces deux coupes ont été recueillies par van Winkle et Okanoto (1953).

Afin de caractériser ces fractions pétrolières, nous avons déterminé les propriétés critiques des pseudo-composés et leurs facteurs acentriques par différentes corrélations. Ceci nous a permis de déterminer l'efficacité de ces dernières et leur influence sur le calcul du flash isotherme.

Le calcul des propriétés critiques a été effectué moyennant les équations de Kesler et Lee (1976) et celles de Cavett (1962). Le facteur acentrique a été calculé quant à lui par l'équation de Thomassen (Pedersen et al., 1989) puis par celle d'Edmister (1958). Nous avons ainsi abouti à quatre caractérisations différentes des deux fractions pétrolières.

En utilisant la règle de mélange conventionnelle et en négligeant les coefficients d'interaction binaires, nous avons appliqué les équations d'état généralisées pour le calcul du flash isotherme. Nous avons ensuite comparé les valeurs des taux de vaporisation obtenus aux valeurs expérimentales données par van Winkle et Okanoto (1953). Les écarts absolus moyens sont donnés dans le tableau 5.

Nous remarquons que les écarts moyens, calculés par rapport aux données expérimentales, varient avec les corrélations utilisées pour le calcul des propriétés. Pour les deux fractions étudiées, les écarts les plus faibles ont été enregistrés avec la première caractérisation. À l'exception de l'équation PRG2 qui n'a apporté aucune amélioration par rapport à celle de PR, les deux autres équations généralisées, à savoir RKG2 et VDWG2, ont quant à elles enregistré des écarts qui sont parfois plus faibles comparés à ceux obtenus avec les équations originelles correspondantes. D'une manière générale, les écarts enregistrés par les équations établies sont du même ordre de grandeur que ceux obtenus par les équations de la littérature.

\section{2 ÉQUATIONS D'ÉTAT MODIFIÉES}

\section{1 Élaboration}

Dans la seconde partie de cette étude, nous avons développé une fonction Alpha pour les deux équations d'état cubiques RK et PR en suivant la même démarche que Soave (1993).

Comme dans le cas des équations généralisées, l'étude a été basée sur les données des $n$-alcanes allant du $\mathrm{C}_{1}$ au $\mathrm{C}_{20}$. Les valeurs d'Alpha ont été calculées à différentes températures réduites et pour différentes valeurs du facteur acentrique. Ce dernier a été calculé en utilisant les valeurs de tension de vapeur calculées par l'équation généralisée de Kesler et Lee (1975), celle de Wagner (1973) ayant donné des résultats peu satisfaisants.

L'intérêt de l'utilisation de l'équation de Kesler et Lee réside théoriquement dans son domaine d'application, qui s'étend du point d'ébullition normal au point critique, et dans sa grande précision s'agissant des hydrocarbures lourds. 


\section{TABLEAU 5}

Écarts absolus en \% obtenus lors du calcul du flash isotherme des fractions pétrolières

Absolute deviations in \% obtained for the calculated isothermal flash of oil fractions

\begin{tabular}{c|c|c|c|c|c|c|c|c|c}
\hline & Caractérisation & RKG2 & TRK & SRK & PRG2 & TPR & PR & VDWG2 & SVDW \\
\hline \multirow{5}{*}{ Fraction pétrolière 1 } & $1^{\text {re }}:$ Kesler-Lee-Pedersen & 14,28 & 14,99 & 15,62 & 14,15 & 14,27 & 12,95 & 14,31 & 16,17 \\
& $2^{\mathrm{e}}:$ Kesler-Lee-Edmister & 34,35 & 36,24 & 37,20 & 33,93 & 34,65 & 32,34 & 34,47 & 38,12 \\
\cline { 2 - 10 } & $3^{\mathrm{e}}:$ Cavett-Pedersen & 15,37 & 15,48 & 15,72 & 15,39 & 16,23 & 14,42 & 15,35 & 16,12 \\
& $4^{\mathrm{e}}:$ Cavett-Edmister & 28,27 & 29,65 & 30,42 & 27,89 & 28,32 & 25,94 & 12,88 & 12,46 \\
\hline \multirow{5}{*}{ Fraction pétrolière 2 } & $1^{\text {re }}:$ Kesler-Lee-Pedersen & 12,94 & 12,49 & 12,40 & 13,08 & 13,08 & 12,36 & 12,88 & 12,46 \\
& $2^{\mathrm{e}}:$ Kesler-Lee-Edmister & 17,81 & 17,88 & 18,15 & 17,79 & 17,89 & 16,77 & 17,81 & 18,41 \\
\cline { 2 - 10 } & $3^{\mathrm{e}}:$ Cavett-Pedersen & 24,66 & 23,74 & 23,09 & 24,82 & 24,54 & 25,82 & 24,61 & 22,69 \\
& $4^{\mathrm{e}}:$ Cavett-Edmister & 18,86 & 18,93 & 19,14 & 18,84 & 18,94 & 17,74 & 18,85 & 19,41 \\
\hline
\end{tabular}

Nous avons ensuite proposé une modification de la fonction Alpha par une extension de l'équation de Soave (1993) :

$$
\begin{aligned}
\alpha & =\left(1+k\left(1-T_{r}^{0,5}\right)\right)^{2}=1+2 k\left(1-T_{r}^{0,5}\right)+k^{2}\left(1-T_{r}^{0,5}\right)^{2} \\
& =1+m\left(1-T_{r}^{0,5}\right)+n\left(1-T_{r}^{0,5}\right)^{2}
\end{aligned}
$$

En exprimant $m$ en fonction de la nature du fluide par le biais du facteur acentrique et $n$ en fonction de $m$ pour chaque hydrocarbure, nous avons calculé $m$ et $n$, puis nous avons établi les équations mathématiques suivantes pour leur détermination :

$$
\begin{gathered}
m=a+b \omega+c \omega^{2}+d \omega^{3} \\
n=e m+f
\end{gathered}
$$

Les valeurs des constantes $a, b, c, d$, $e$ et $f$ sont données dans le tableau 6.

\section{TABLEAU 6}

Valeurs des paramètres des équations de Redlich-Kwong (RKM) et Peng-Robinson (PRM) modifiées

Values of the parameters of modified Redlich-Kwong (RKM) and Peng-Robinson (PRM) equations

\begin{tabular}{c|c|c}
\hline & RKM & PRM \\
\hline$a$ & 1,0183736 & 0,78038497 \\
$b$ & 2,955939 & 2,7414309 \\
$c$ & $-0,088633814$ & $-0,067899189$ \\
$d$ & $-0,11727599$ & 0 \\
$e$ & 1,0329673 & 1,0274425 \\
$f$ & $-1,126727$ & $-0,71468581$ \\
\hline
\end{tabular}

La courbe de la figure 2 justifie bien la linéarité du paramètre $n$ en fonction de $m$ de l'équation (12).

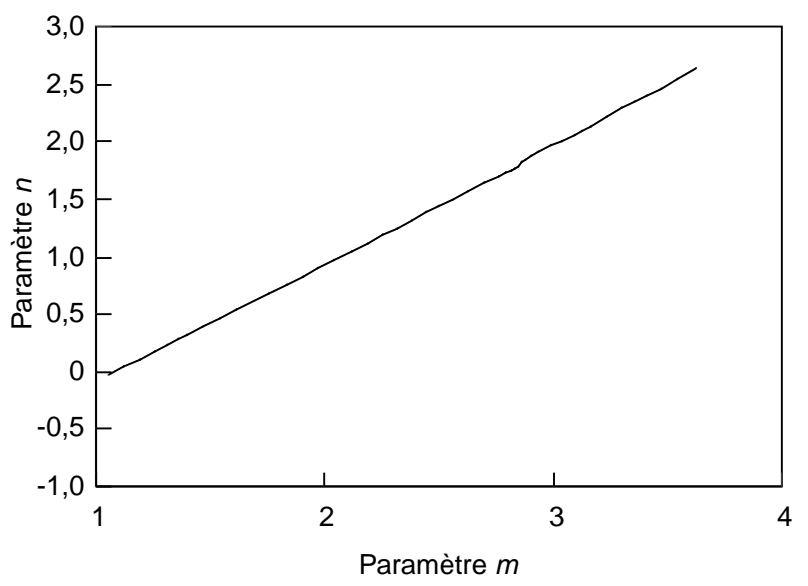

Figure 2

Variation du paramètre $n$ en fonction du paramètre $m$ dans le cas de l'équation de Redlich-Kwong modifiée.

Variation of parameter $n$ in function of parameter $m$ in the case of the modified Redlich-Kwong equation.

\subsection{Application aux hydrocarbures purs}

De la même manière que pour les équations généralisées, les équations modifiées ont été appliquées au calcul des propriétés thermodynamiques des hydrocarbures purs. Les résultats obtenus pour la tension de vapeur sont acceptables et se comparent favorablement aux données de référence. Bien que les équations d'état modifiées aient été établies à partir des données de tension de vapeur des $n$-alcanes, elles ont été appliquées avec succès aux hydrocarbures de structure chimique différente. 
TABLEAU 7

Écarts absolus obtenus pour le calcul de la tension de vapeur

Absolute deviations obtained for the calculated vapour pressure

\begin{tabular}{l|c|c|c|c|c|c}
\hline HC & Nombre & Points & RKM & SRK & PRM & PR \\
\hline$n$-alcanes & 22 & 1334 & 2,99 & 2,80 & 2,70 & 11,72 \\
Alcanes ramifiés & 45 & 2164 & 2,49 & 1,89 & 2,20 & 2,26 \\
Alkylbenzènes & 29 & 1717 & 4,36 & 5,35 & 4,10 & 9,47 \\
Alkylcyclopentanes & 16 & 737 & 2,45 & 2,34 & 2,40 & 2,67 \\
Alkylcyclohexanes & 10 & 592 & 16,23 & 16,97 & 16,36 & 27,60 \\
Moyenne & 122 & 6545 & 4,22 & 4,40 & 4,10 & 8,42 \\
\hline
\end{tabular}

TABLEAU 8

Écarts absolus obtenus pour le calcul des propriétés des mélanges

Absolute deviations obtained for the calculated properties of mixtures

\begin{tabular}{|c|c|c|c|c|c|}
\hline Mélange & Propriété & SRK & RKM & PR & PRM \\
\hline \multirow{3}{*}{$\mathrm{C}_{1}-\mathrm{C}_{4}$} & $P_{b}$ & 13,58 & 7,07 & 13,99 & 11,78 \\
\hline & $V^{L}$ & 26,69 & 9,68 & 19,85 & 15,42 \\
\hline & $V^{L}$ translaté & 29,84 & 9,56 & - & - \\
\hline \multirow{3}{*}{$\mathrm{C}_{1}-\mathrm{C}_{10}$} & $P_{b}$ & 16,04 & 9,84 & 17,11 & 14,40 \\
\hline & $V^{L}$ & 19,64 & 19,11 & 6,65 & 6,21 \\
\hline & $V^{L}$ translaté & 17,84 & 17,18 & - & - \\
\hline \multirow{3}{*}{$\mathrm{C}_{3}-\mathrm{C}_{5}$} & $P_{b}$ & 2,52 & 2,81 & 3,35 & 3,41 \\
\hline & $V^{L}$ & 10,70 & 10,12 & 4,28 & 4,35 \\
\hline & $V^{L}$ translaté & 10,33 & 9,76 & - & - \\
\hline
\end{tabular}

TABLEAU 9

Écarts absolus obtenus lors du calcul du flash isotherme des fractions pétrolières

Absolute deviations obtained for the calculated isothermal flash of oil fractions

\begin{tabular}{|c|c|c|c|c|c|}
\hline & Caractérisation & RKM & SRK & PRM & PR \\
\hline \multirow{4}{*}{ Fraction pétrolière 1} & $1^{\text {re }}:$ Kesler-Lee-Pedersen & 16,51 & 15,62 & 16,06 & 12,95 \\
\hline & $2^{\mathrm{e}}:$ Kesler-Lee-Edmister & 38,27 & 37,20 & 37,83 & 32,34 \\
\hline & $3^{\mathrm{e}}:$ Cavett-Pedersen & 16,57 & 15,72 & 16,23 & 14,42 \\
\hline & $4^{\mathrm{e}}:$ Cavett-Edmister & 31,99 & 30,42 & 31,34 & 25,94 \\
\hline \multirow{4}{*}{ Fraction pétrolière 2} & $1^{\text {re }}:$ Kesler-Lee-Pedersen & 16,51 & 12,40 & 11,91 & 12,36 \\
\hline & $2^{\mathrm{e}}:$ Kesler-Lee-Edmister & 38,27 & 18,15 & 18,41 & 16,77 \\
\hline & $3^{\mathrm{e}}:$ Cavett-Pedersen & 22,14 & 23,09 & 22,33 & 25,82 \\
\hline & $4^{\mathrm{e}}:$ Cavett-Edmister & 19,49 & 19,14 & 19,23 & 17,74 \\
\hline
\end{tabular}


Le tableau 7 montre que les écarts absolus moyens enregistrés par l'équation RKM n'ont pas été améliorés comparativement à l'équation SRK, alors que l'équation PRM a fourni des écarts qui sont nettement inférieurs à ceux obtenus avec l'équation PR. En effet, pour cette dernière, les écarts ont parfois été réduits de plus de moitié.

\subsection{Application aux mélanges et fractions pétrolières}

Nous avons appliqué les équations d'état modifiées aux mélanges et aux fractions pétrolières de la même manière que pour les équations généralisées. Nous remarquons une nette diminution des écarts dans la prédiction des propriétés thermodynamiques des trois mélanges étudiés, alors que pour les fractions pétrolières, nous n'avons enregistré aucune amélioration, indépendamment de la méthode de caractérisation utilisée. Les résultats obtenus sont consignés dans les tableaux 8 et 9.

\section{CONCLUSION}

Partant de l'équation d'état cubique de van der Waals, nous nous sommes basés sur les modifications les plus récentes, à savoir les équations de Sator et Chitour (1998), afin d'élaborer de nouvelles équations d'état généralisées.

Notre seconde approche a consisté à modifier la fonction Alpha des équations d'état de Redlich-Kwong et PengRobinson sans avoir recours à la loi des états correspondants.

L'application des équations d'état ainsi obtenues aux hydrocarbures purs a permis d'améliorer la prédiction des propriétés thermodynamiques. Leur application aux mélanges a également engendré des résultats positifs, ce qui nous a encouragés à les appliquer aux fractions pétrolières. Pour ces dernières, les résultats obtenus sont en accord avec les valeurs expérimentales et sont en général du même ordre de grandeur que ceux obtenus par les équations d'état SoaveRedlich-Kwong et Peng-Robinson.

\section{RÉFÉRENCES}

Cavett, R.H. (1962) 27th Mid-Year Meeting of the API Division of Refining, San Francisco, USA.

Design Institute for Physical Properties Data (DIPPR) (1995) AIChE J.

Edmister, W.C. (1958) Pet. Refin., 37, 173.

Jan, D. et Tsai, F. (1991) Canadian Journal of Chemical Engineering, 69, 992-996.

Kesler, M.G. et Lee, B.I. (1975) AIChE J., 21, 510.

Kesler, M.G. et Lee, B.I. (1976) Hydrocarbon Processing, 3, 153.

Pedersen, K.S., Thomassen, P. et Fredenslund, A. (1989) Properties of Oils and Natural Gases, Gulf Publishing Company, 5, Houston, USA.

Péneloux, A., Rauzy, E. et Frèze, R.A. (1982) Fluid Phase Equilibria, 8, 7-23.

Peng, D.Y. et Robinson, D.B. (1976) Ind. Eng. Chem. Fundam., 15, 59.

Perry, M.S. et Chilton, J.C. (1990) Chemical Engineering Hand Book, McGraw Hill, New York.

Raznnjevic, S. (1976) Hand Book Thermodynamics Tables and Charts, Addison Wesley, New York.

Redlich, O. et Kwong, J.N.S. (1949) Chem. Rev., 44, 233.

Reid, R.C., Prausnitz, J.M. et Poling, B.E. (1988) The Properties of Gases and Liquids, $4^{\mathrm{e}}$ édition, McGraw Hill, New York.

Sage, B.H., Budencholzer, R.A. et Lacey, W.N. (1940) Ind. Eng. Chem., 9, 1262.

Sator, S. et Chitour, C.E. (1998) Fluid Phase Equilibria, 153, 73-80.

Soave, G. (1972) Chem. Eng. Sci., 27, 1197-1203.

Soave, G. (1984) Chem. Eng. Sci., 39, 357.

Soave, G. (1993) Fluid Phase Equilibria, 84, 339.

Twu, C.H., Coon, J.E. et Cunningham, J.R. (1994) Fluid Phase Equilibria, 96, 19-31.

Twu, C.H., Coom, J.E. et Cunningham, J.R. (1995) Fluid Phase Equilibria, 105, 61-69.

Van der Waals, J.D. (1873) Doctoral Dissertation, Leiden, Hollande.

Van Winkle, M., Okanoto, K.K. (1953) Ind. Eng. Chem., 45, 2, 429-439.

Wagner, W. (1973) Cryogenics, 13, 470.

Manuscrit final reçu en mai 2001 